\title{
REVIEW
}

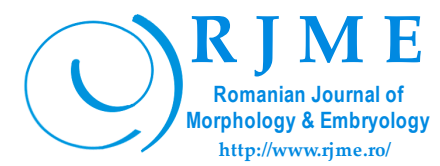

\section{Morphopathological approaches in alcoholism}

\author{
Ana Miruna Drăgoi ${ }^{1)}$, Tiana Voicu ${ }^{2)}$, Ana Maria ChipeşiU²), Radu Virgil Costea ${ }^{3)}$ \\ 1) Department of Psychiatry, Prof. Dr. Alexandru Obregia Clinical Hospital for Psychiatry, Bucharest, Romania \\ 2) Faculty of Psychology and Educational Sciences, University of Bucharest, Romania \\ ${ }^{3)}$ Department of General Surgery, Carol Davila University of Medicine and Pharmacy, Bucharest, Romania
}

\begin{abstract}
Alcohol morphopathology has been studied over time, being a central interest of specialists, due to the negative consequences it has on the brain and the entire central nervous system (CNS). This paper is a review of the literature that emphasizes one of the problems of the modern world, that of the compulsive consume of alcohol, having a great global spread. The studies analyzed are topical, being carried out in recent years and consider the harmful effects of alcohol on brain formations, such as corpus callosum, gray and white matter, hippocampus, and hypothalamus. At the same time, alcohol is a risk factor for cardiovascular diseases, and in combination with other harmful substances, increases the risk of various diseases, such as neurodegeneration. Abusive alcohol consumption can bring epigenetic changes and alter the typical functioning of cognitive functions. This paper focuses on alcohol consumption on adolescents and young people, which is a serious problem nowadays. Alcohol also influences the way of behavioral expression, becoming a risk for the development of mental disorders. However, alcohol withdrawal is another problem with different effects and must be in the attention of specialists.
\end{abstract}

Keywords: alcohol, gray matter, white matter, hippocampus, neurodegeneration, neuroinflammation.

\section{口 Introduction}

Alcoholism occurs through a combination of several factors. Various biological factors are included here, where a wide range of genetic changes or abnormalities are also included. At the same time, it can be considered nongenetic causes, such as dysfunctional social relationships, neuroadaptive responses or interaction with the environment. The risk for alcoholism is related to the family history, the environment in which the individual developed, but especially it is about adverse events in childhood. The combination of these factors gives rise to complex relationships and involves overlaps and effects of many genes that affect brain development, structure and function, the way they process information when it comes to alcohol consumption, and sensitivity to it [1].

Assiduous intensive research work involving international collaboration and advanced technology favored the discovery of genetic variations and biomarkers that occur in the issue of alcoholism and its evolution. The study of Manzardo et al. (2015) identified 337 genetic biomarkers relevant to alcoholism. They can be causal or transient intersecting with the way body responds to alcohol consumption and with environmental factors, such as a disorganized lifestyle (drug use, diseases, or viruses specific to alcohol consumption) [1].

One of the most serious and critical problems in most countries is excessive alcohol consumption. Separately, in Europe, one of the largest alcohol markets and also one of the most famous regions where alcohol is consumed, about 60 million citizens have problems with alcohol, and 23 million suffer from alcohol dependence [2]. Thus, problems caused by alcohol are some of the most common neuropsychiatric problems affecting society nowadays. Research in the fields of clinical and neuroimaging, aimed to study the issue of alcohol consumption, has found that the involvement of prefrontal-striatal connectivity can promote addictive behavior. The use of so-called 'omics' technologies is widely used to identify molecular alterations. This term refers to a set of procedures that allows the simultaneous evaluation of hundreds of species of molecules from cells or tissues. In conjunction with advanced bioinformatics technology, these applied methods help to comprehensively understand the overall response of organisms to various viruses, diseases, or drugs [2].

\section{ㅁ Neuropathology of alcoholism}

The innate immune response to the central nervous system (CNS) is responsible for both synaptic plasticity and neuronal damage. The evidences gained over time from animal and human studies show how important is the role of the neuroimmune system in the activity of ethanol (EtOH) on CNS. Research has shown that alcohol stimulates brain immune cells, microglia, and astrocytes by innate activation of Toll-like receptors (TLRs) and nucleotidebinding oligomerization domain (NOD)-like receptors (inflammasome NLRs) that trigger signaling pathways that result in neuroinflammation. The CNS can recognize and transmit an immune response to pathogens or toxins. Inflammatory responses against pathogens are initiated by receptor recognition molecules that detect molecular pathways associated with pathogens [3].

Alcoholism is a serious health problem that involves neurodegradation. Alcohol consumption combined with genetic factors, diseases, drugs can cause intracellular changes in neuronal tissue. What degrades the CNS and one of the most harmful factors is the oxidative stress caused by alcohol. Previous research suggests that the endogenous antioxidant system is one of the most important

This is an open-access article distributed under the terms of a Creative Commons Attribution-NonCommercial-ShareAlike 4.0 International Public License, which permits unrestricted use, adaptation, distribution and reproduction in any medium, non-commercially, provided the new creations are licensed under identical terms as the original work and the original work is properly cited. 
systems in the brain tissue. Excess or lack of antioxidants in chronic alcoholics leads to the sensibilization of neurons resulting in their destruction and the beginning of the neurodegradation process [4].

Abusive drinking is the third ranked factor causing some diseases because of its devastating effects on the brain. In addition to all this, alcohol consumption comes with a set of comorbidities, like liver disease. Cirrhosis, in particular, often leads to hepatic encephalopathy, which represents a glial disease [5].

Hepatic encephalopathy is a neuropsychiatric disorder that manifests itself through cognitive difficulties, confusion, delirium and even decreased coordination for fine movements. This type of condition occurs because of liver dysfunction, associated with cirrhosis, being caused by prolonged alcohol consumption. In the same line as liver dysfunction, hepatic myelopathy, which mainly attacks the spine, may occur. This can be manifested by bilateral spastic paraparesis and lower limb hyperreflexia [6].

One of the important organs that are affected because of alcohol abuse is the liver. His major biological function is to stop the transmission of toxic effects followed by alcohol ingestion, which have harmful consequences in the brain and the body as a whole. In this way, the poor and defective functioning of the liver leads to a lack of defense of the body in the face of physiological deficiencies. Most alcoholics experiment cerebral atrophy, a reduction in the volume of white matter and gray matter, especially around the hypothalamus and thalamus [6].

\section{ㅁ Alterations in brain formations}

In brain research, the most common omics approaches to date are transcriptome profiling on animal models or on postmortem brain samples. Through these methods, new mechanistic perspectives have been discovered, but when it comes to these methods applied to mental disorders, the power of the transcriptome is usually low [2]. The study conducted by Meinhardt et al. [2] offers specialized literature some interesting new perspectives. The authors suggest that distinct regions of the brain, even very close ones (such as infralimbic or prelimbic) can be separated based on their metabolic phenotypes. The same authors suggest that alcohol consumption profoundly alters neurometabolic profiles, especially in circuits that are known to have a sensitivity to the long-term effects of alcohol, like infralimbic-accumbens shell circuit. The concentration of regional tissue of certain metabolites (in particular, dopamine and Met-enkephalin) may allow extrapolation of the recent past of alcohol consumption. At the same time, the aforementioned authors suggest that the identification of a metabolic fingerprint that persists longer than the current period of consumption might indicate an unknown pathophysiological mechanism in alcohol dependence [2]. Global metabolic profiling by mass spectrometry is a feasible way to study metabolome alterations in postmortem brain tissue and is an extremely useful extension for omics technologies used to decipher neuropsychiatric pathologies, including those related to alcohol addiction [2].

The possibility of detecting brain conditions caused by alcohol consumption has been demonstrated through research of neurometabolomic markers. This kind of data indicate major changes in the infralimbic-accumbens shell pathway. Through clinical perspective, positron emission metabolic tomography can identify altered metabolism in different regions of the brain [2].

Abusive alcohol consumption can lead to inflammatory processes in the hippocampus and cerebellum. These types of degenerative processes at the neurological level are seen as having a potential cause in the action of micro-ribonucleic acid (miRNA), in particular through the action of the miR155 derivative. It seems that the possibility of stopping the action of this miRNA has a therapeutic effect on improving these inflammatory processes caused by alcohol ingestion and requires the attention of specialists [7].

These neuroinflammatory processes occur because of signals received by the brain at the time of alcohol abuse. The alarm signal is given by cells fighting to preserve the immunity of the CNS, such as glial cells and TLRs. These types of receptors produce an activation of glial cells, which ultimately leads to the development of such inflammation or neurodegeneration [8]. TLRs are the first identified and best characterized receptors discovered, being a primary defender for microbial infections. The TLR family comprises 10 members for human body (from TLR1 to TLR10), but the most important is TLR4, which is the receptor that recognizes lipopolysaccharides (LPS), molecules that exists in the outer part of Gram-negative bacteria. NLRs have a cytoplasmic nature and they fight to detect pathogens, enabling an immune response [3]. The data suggest that overactivation of TLR4 or NLRs causes neuronal damage in the cerebral cortex and hippocampus, while modest activation of TLR4 modulates neurotransmission, alcohol consumption, and alcohol rewards. Lack or elimination of TLR4 and NLR family pyrin domain containing 3 (NLRP3) abolish many neuroimmune effects of EtOH [3].

The same observations were also captured in a similar study, which analyzed the components of the cerebral cortex of alcoholics. In this respect, it seems that TLRs, especially TLR7, performs an activation in brain structures like the hippocampus, and thus leads to the neuropathologies of alcoholism. This activation produces the release of glial cells, which is also claimed by postmortem investigations that capture some disturbances in their population [9].

The nucleus accumbens (NAc) is a neurological formation with an important role in the compulsive consumption of alcohol, especially in cases of addictions. Since the neurons specific to this formation are specialized in formulating reward behavioral responses, it seems that stopping their stimulation may become essential in treating EtOH addiction. This inhibition of the motivation to seek the fulfillment of the pleasure created by the addiction is achieved by the opposite action of the dopaminergic population like medium spiny neurons (MSN) through D2 receptors, as opposed to D1 [10].

\section{무 Cloninger typology of alcoholism}

Alcohol abuse was classified, according to Cloninger $[11,12]$, as being divided into two typologies, depending on the age of the start of consumption. Thus, typology 1 occurs in people over 25 years old, and typology 2 is characteristic of people under 25 years of age. The first 
typology is defined by a consumption behavior learned in the form of negative conditioning, which leads to a susceptibility to anxious behavior. Unlike these people, those in typology 2 acquire consumption through positive conditional learning, being rather characterized by impulsiveness and even antisocial behaviors. It appears that this type of conditioning has a biological basis, also involved in supporting addictions, namely the $\alpha$-amino3-hydroxy-5-methyl-4-isoxazolepropionic acid (AMPA) receptors. Their increase in density has been reported in the synapses of people in typology 2 , especially in the anterior cingulate cortex (ACC). This suggests poor functioning of the ACC, so decision-making and rewardassignment processes are altered [13].

\section{ㅁ Alcohol-induced dementias}

Neurodegradation is a serious consequence in chronic alcoholics that can occur in the form of dementia or brain damage from alcohol addiction. Degradation refers to changes related to the plasticity of the brain that underlie alcohol dependence, tolerance, and withdrawal effects. The neuronal changes that occur because of repeated alcohol consumption differ from other diseases, such as Alzheimer's in which, although the brain atrophies, the neuronal losses are modest. Moreover, brain recovery from abstinent alcoholics is unique among these diseases. Postmortem brain tissue is an important resource for further research into the problem of alcoholism. At autopsies in individuals with brain damage caused by alcohol consumption, it was observed that brain atrophy is regionally specific, but mostly observed around the frontal lobes. Brain atrophy is specific to a particular region and is closely related to white matter loss. The mild atrophy that occurs in gray matter results mainly from the withdrawal of the neuronal dendritic tree. Chronic alcohol consumption has serious and varied effects on the brain and involves damage to cell membranes, damage to the blood-brain barrier and increased oxidative stress [14].

Korsakoff syndrome is a type of disorder based on cognitive difficulties, especially at the level of memory functions. This syndrome is often also associated with chronic alcohol consumption, leading to temporal confusion and disorientation. In order to be able to distinguish between the two types of conditions, the neurological and cognitive consequences on some patients have been studied by means of a psychological sample of continuous recognition of some of the elements presented. The purpose of these investigations was to determine the basic source of memory difficulties arising from such disorders. In the case of Korsakoff syndrome, the presence of memory disorders based on temporal context, in the form of confabulations, is noted. These types of memory deficits are not also noticeable in the case of chronic alcohol consumption. Following the results of the study cited, there is no continuum between the two types of neurological conditions, so chronic alcohol consumption is not characterized by a specific source of memory deficits and not always leads to the syndrome [15].

The psychological sample used in the above-mentioned study becomes a useful tool in making a differential diagnosis and especially to be able to capture the risk factors towards the further development of Korsakoff syndrome. Although chronic alcohol consumption does not exhibit specific memory disorders, following a detoxification treatment, these individuals are at risk of developing clinical symptoms specific to the syndrome [15].

Wernicke's encephalopathy is an acute neuropsychiatric disorder caused by lack of absorption of vitamins, especially thiamine, in alcoholics, but not only. It manifests itself through ataxia, ophthalmoplegia and leads to alteration of psychic capacities. In the case of this disorder, there is a continuum at the end of which the Korsakoff syndrome is found. In this respect, a person who consumes alcohol abusively may end up developing Korsakoff syndrome, which is in fact the chronic variant of Wernicke's encephalopathy. Both of these conditions can lead to sudden death as a result of damage to cardiorespiratory centers in the brain [6].

\section{ㅁ Alcohol and cerebrovascular dysfunction}

Moderate alcohol consumption has a beneficial effect on the body, which can serve as a protective factor on cardiovascular diseases and vascular or hemorrhagic accidents. On the contrary, prolonged consumption above the moderate limit becomes a risk factor for all of the above-mentioned conditions. Alcohol poisoning or excessive consumption in a short period of time can induce a heart attack, this being caused by increased blood pressure, which can lead to the placement of a blood clot on the vessels. Increased levels of homocysteine (Hcy) amino acid, along with epigenetic changes caused by alcohol abuse, are seen as factors that increase the risk of developing cardiovascular disease, vascular dementia or stroke [16].

\section{a Neurophysiological mediators of disorders caused by alcohol consumption}

Thiamine, also known as vitamin B1, plays an important role in the swelling and conditions caused by alcohol consumption. As a result of this consumption, a difficulty in absorption and use of this vitamin occurs which leads to a deficiency in the body. People suffering from a thiamine deficiency may experience disturbances in spatial memory and behavioral disorders. The effect of chronic alcohol consumption is added to this deficiency, sometimes preexisting and contributes to the loss of neurons plasticity, which causes progressive cognitive dysfunction. At the same time, along with an abusive alcohol consumption, thiamine deficiency causes a demyelination of the brain components, resulting in a low volume of white matter. Although this type of vitamin appears to play a crucial role in alcohol-related disorders, however, specialists show that simply trying to reduce deficiency in the body is not enough to treat brain neuropathologies [6].

Recent studies bring to the fore the potential therapeutic effects of some cells in the body, which require increased attention regarding neurodegenerative disorders of the CNS. Recently, erythropoietin (EPO), with a protective role in such disorders, also caused by abusive alcohol consumption, have been discovered. These proteins regenerate synapses intoxicated by substance consumption in the cortex and hippocampus. However, it takes an extended period of time for the restored structures to become stable and strong, like the initial ones [17]. 


\section{alcohol and its effects on the brain in adolescents}

Data from recent years indicate that the brain is following its natural course of development during adolescence and undergoes not only important structural but also functional changes at this stage of development. These changes in the functional maturation of the brain, correlated with the individual regions of the brain, are necessary for cognitive success, motor, and sensory functions from childhood to adulthood. Changes in the prefrontal cortex and subcortical structures (e.g., the medial temporal lobe, amygdala, hippocampus, NAc) are the most important areas in the remodeling process. A multitude of studies have shown that the adolescent's brain is prone to devastating effects caused by EtOH. Alcohol consumption, a common practice among adolescents, is responsible for many structural and functional changes in their brains. These changes are associated with long-term cognitive dysfunction and loss of behavioral flexibility [3]. In addition to these changes, there is also neuroinflammation in the adolescent's brain. According to animal studies, overexpression of high mobility group box 1 (HMGB1) and TLRs has been observed in the orbital frontal cortex. These expressions are found also in the human brain, where these have been associated with pathological alcohol consumption [3].

Adolescence is a critical period of development in which various structural and functional changes in synaptic plasticity and neural conceptuality occur. These changes consist of two cellular processes: myelination and synapse removal. Those processes are important in order to maintain the health of the brain and effective communication between brain regions. Some experimental clinical trials suggest that the adolescent brain is sensitive to the toxicity of alcohol. Alcohol consumption by adolescents has negative effects on white matter, reduces the tracts between myelin fibers and frontal connections, all of which in turn underlie the dysfunction of cognitive learning skills, memory, and executive functions [18].

An important consequence of continuous alcohol consumption in adolescence is the danger of developing and maintaining addiction throughout adulthood. The adolescent period is characterized by a series of behaviors conducive to risk-taking. These characteristics are understood through changes at the hormonal level, mainly those associated with stress, that may explain the propensity to consume toxic substances. Development of synaptic functions and neural structures in the brain of adolescents are considered to be important processes for a cognitive phenotype similar to that of adults. This period characterized by an increased neuroplasticity offers a bigger vulnerability to actions that involve addictions. Degradation of the mesocorticolimbic dopamine pathway in addition with EtOH-induced neuroinflammation and myelin modification during adolescence period could underlie cognitive dysfunction and predispose to alcohol dependence [18].

\section{ㅁ Alcohol and gender differences}

Alcoholism is most often associated with the pathology of gray and white matter. Neuroimaging showed reductions in brain volume and alcohol-related axonal degradation. A 2013 study analyzed the consequences of alcoholism on the amount of white matter in lobar and gyral subdivisions of the brain. The study had 42 abstinent alcoholics and 42 individuals as control participants. They also examined the white matter of the cerebellar and regions of the corpus callosum. The authors also evaluated the links between ventricular volumes and white matter with drinking patterns and gender differences as well. The authors found differences between the volume of white matter in alcoholics group compared to the control group, especially in the corpus callosum, but also strong differences between men and women [19].

Abusive alcohol consumption for many years has a toxic effect on the frontal and temporal white matter in women, whereas in men on the corpus callosum [19]. Alcoholic women have a small corpus callosum and larger ventricular volumes, while alcoholic men have a larger corpus callosum. Data indicates that in the brain of alcoholic women are connections between the period of sobriety and the volume of white matter, in comparison to men. Therefore, the consequences of alcohol consumption on white matter vary according to gender. Alcoholic women show the highest sensitivity in the frontal, temporal, ventricular regions, and corpus callosum, while in alcoholic men the main effects are in the corpus callosum. White matter recovery occurs faster in women, as opposed to men [19].

Some research has shown that the reward network for alcoholic men is lower than non-alcoholic men, but data on alcoholic women are little researched. Morphometric data were performed on brain scans of 60 chronic alcoholics and 60 non-alcoholics as a control group. The relationships between volume data and the history of alcohol abuse were examined. Analyzes indicate that alcoholic men have lower volumes of rewards than non-alcoholics, and alcoholic women have higher volumes of rewards than non-alcoholics. Previous findings show that there are significant differences between men and women in terms of alcoholism. The data suggest that there are differences in the reward circuit in the brain between men and women [20].

At the same time, the women life is highly influenced by progesterone, that appears after ovulation and in postpartum syndromes, alters moods, leading up to depression or circadian rhythm disorders [21]. Linked to possible genetic in heritage or predisposition to a non-healthy life, leads sometimes to depression and this is a close step to uncontrolled alcohol consumption.

\section{$\neg$ Neuroimaging changes}

Alcohol abuse over time becomes a risk factor for brain disorders that may vary in intensity. Encephalopathy is a type of dysfunction with increased severity, several forms of it being known. Postmortem imaging investigations show changes in the neurological system, accompanied by a degeneration of the corpus callosum, especially in rare disorders, such as Marchiafava-Bignami syndrome. The evolution of neuroimaging allows the revelation of cortical lesions, having an important role in establishing a prompt diagnosis and prevention in cases of chronic alcohol consumption. In this way, especially in the case of the abovementioned syndrome, intervention treatments focusing on increasing the ability to transmit nutrients, such as thiamine, through the blood--brain barrier can be established. 
Based on computed tomography (CT) or magnetic resonance imaging (MRI) medical examinations, changes can be tracked to prevent potential necrosis of some brain component units. In this respect, it is recommended to use symptomatic treatments to avoid major degradation of the nervous system, which can lead to sudden death in case of acute poisoning [22].

Clinical studies in neuroimaging are particularly relevant for highlighting the impact of alcohol abuse at the neurological level. There are special clinical cases that bring into account diseases that occur at the level of the brain, which are not accompanied by other types of disorders. In this way, a physiological deterioration of the brain can lead to the inability to maintain the position of the body, tremor in the arms and even a form of nystagmus. However, cognitive functions continue to be preserved [23].

\section{a Genetic transmission of alcoholism - epigenetics}

EtOH dependence leads to epigenetic changes, which occur particularly in histone proteins and ultimately in the deoxyribonucleic acid (DNA) component. In a study conducted, the processes of histone deacetylase in the brain were identified because of alcohol consumption. These results reveal changes in the way genes are expressed, especially in the region of the cerebral cortex and in the hippocampus. However, there cannot be a generalized change, as it appears that the two regions concerned are influenced differently by a certain population of cells prevailing in each region. This study highlights the need to study epigenetic changes that may be useful in the treatment of alcohol dependence [24].

Previous studies have identified alcohol-responsive brain networks through a methodology of case-control in which some individuals that were family related were studied based on whole-genome approach. Also, some of them were alchoholics and the rest were not. Candidate gene strategies consider the neurobiological pathways and neurodevelopment key to brain function through the biochemical rewards related with the psychological effects of alcohol, mostly those that affect metabolic processes. Moreover, biomarkers at the genetic level, including chromosomal regions, were identified by combining genetic and functional data with phenotypes of alcoholism, all to identify the molecular signature of alcoholism phenotype [1]. An example of this sort of analysis is the alcohol dehydrogenase $(A D H)$ enzyme, which strongly contributes to the metabolism of alcohol. This biomarker is related to the family of $A D H$ genes located in the 4q23 chromosomal band. This chromosome (4q23) has been strongly associated with vulnerability to alcoholism in several studies. The activity of enzymes that metabolize alcohol (e.g., ADH) has effects on sensitivity to alcohol, its effects on the body being toxic. Some genetic alternative forms that modify the processing of alcoholic intercessor (e.g., ADH1B, ADH1C, ALDH2) impact the probability of generating a deviation in alcohol consumption [1].

At the same time, there are strong correlations between alcohol and schizophrenia sometimes due to alcoholic hallucinosis - a schizophrenia-like psychosis, sometimes impossible to be distinguished from schizophrenia itself.
More than that, the dopamine receptor D3 (DRD3) gene is considered to be involved both in schizophrenia, alcohol and drug abuse and addiction [25].

\section{a Abstinence and withdrawal of alcohol consumption}

A reference study [26] considers neurological changes that occur in both the human and animal species, at the time of compulsive alcohol withdrawal. The basic hypothesis is to observe reductions in the level of gray matter, following hyperglutamatergic metabolism. In this respect, in the early stages of abstinence, the volume of gray matter decreases, at the same time as the increase in glutamate makers. Also, it appears that metabolic neuronal cells [N-acetylaspartate (NAA)], although affected by alcohol abuse, recover immediately after withdrawal. The same study shows that there is also a decrease in the volume of the hippocampus, at the same time as the increase in glutamate levels, but these changes do not occur until several weeks after abstinence. The result with an impact on medical practice, and not only, is precisely this change, which suggests that neuronal degradation occurs during the period of alcohol withdrawal and not during actual consumption [26].

Due to the dependency linked by alcohol consumption, in which the body and the brain gets involved, the withdrawal can be difficult without proper medication (like benzodiazepines and seizure medication), considering at least the side effects, like shaking, insomnia, anxiety, nausea, going up to delirium tremens and personal determination for this step [27].

\section{a Cumulative effects of alcohol and tobacco}

Chronic alcohol consumption leads to neurodegradation and impact the cognitive functions. Some past studies from pathological and neuroimaging domains have highlighted that the main cause of brain atrophy in alcoholics is the white matter loss. Consequently, some neuronal losses appear and are found in certain regions, such as the prefrontal cortex. Studies conducted on cigarette smokers also indicate that tobacco smoke can determine brain atrophy through inhalation. Given that most alcoholics are also smokers, it has been assumed that some brain regions that are damaged because of alcohol can be also damage by tobacco use [28].

Tobacco and alcohol are the most used substances and represent a danger for developing health problems. A considerable number of researches show that alcohol degrades the cells of the brain and can lead to impaired cognitive functions and inability to solve problems. One of the main causes of brain volume reduction is also related to the loss of white matter. Chronic smoking has been associated with impairments in executive function, general intellectual abilities, learning or memory, and cognitive inflexibility. Regarding tobacco use, it is still unknown if it has effects or not on atrophy of the brain, or if there are real changes in white matter volume [28].

The results of a 2017 study [28] suggest that there is a synergistic effect between the two exposures to tobacco or alcohol in terms of myelinated fiber density, but more complex interactions with other white matter indices. 
Tobacco affects mostly the myelin, while EtOH seems to target the axons in the white matter. The authors found that there was a minimal difference in the way EtOH acts on the brains of rats compared to nicotine. As mentioned above, alcohol does not target the myelin thickness, but like nicotine, it influences fiber density. The results of the study provide evidence that alcohol and nicotine result in white matter degeneration. Nicotine and EtOH share some effects about their pathomechanisms, as seen on indices measurement, but nicotine showed a bigger effect on myelin integrity and EtOH had an additional effect on axons [28].

The cumulative effects of alcohol and nicotine led to degenerative marks at the white matter level, causing reductions in glycoprotein expression that is associated with myelin. Exposure to tobacco and alcohol consumption favor neuronal degradation by affecting the synthesis, maturation, and integrity of myelin by distinct but overlapping mechanisms [29].

\section{다이 Lifestyle neuropathology: how our behavior harms our brain and what can we do about it}

The most common neurological disorders (e.g., Parkinson, Alzheimer, stroke, or brain damage) are not the result of unfavorable genetics. Most mental illnesses, such as Alzheimer's disease and stroke, are strongly influenced by lifestyle (smoking, obesity, education, physical and social activity). There are other harmful activities that can lead to brain damage, and here we are talking about alcoholism, drug use, exposure to toxic substances, chronic stress that have negative effects on the normal functioning of the brain. Lifestyle choices can greatly reduce the risk of developing a mental illness. It has been estimated that $50 \%$ of all Alzheimer's disease can be prevented by reducing risk factors, such as smoking, and by enhancing physical and mental activity. It also adds, of course, the treatment and prevention of chronic diseases in time. Similarly, $80 \%$ of strokes can be prevented by changing lifestyle and making healthy decisions [30]. At the same time, alcoholism can hide a depressive pathology but is linked to patient's determinism and sometimes with the abandonment of the treatment. Motivations can also be linked to the burn-out syndrome with various pathology, seen as a depressive symptom, from working conditions and tasks up to neuroendocrine changes [31].

\section{a Conclusions}

Alcohol consumption affects, in principle, almost any type of process of the organism. The base substance is rapidly absorbed and is transported through the blood, passing through the bathing of the membranes. Its effects are harmful to people's brains, whether it is developed or developing. The main effect of alcohol on the body is a depressor one, which often changes the expression of behavior and alters cognitive functions. Alcohol is a main risk factor for the development of various CNS disorders, which can cause dementia or even strokes, even leading to sudden deaths in case of excessive consumption. It also has an implication in the expression of genes and can have a cumulative effect with that of other types of substances (e.g., nicotine), being as a whole harmful to the body, especially on the brain.

\section{Conflict of interests}

The authors declare that they have no conflict of interests.

\section{References}

[1] Manzardo AM, McGuire A, Butler MG. Clinically relevant genetic biomarkers from the brain in alcoholism with representation on high resolution chromosome ideograms. Gene, 2015, 560(2):184-194. https://doi.org/10.1016/j.gene.2015.01.064 PMID: 25655461 PMCID: PMC6675570

[2] Meinhardt MW, Sévin DC, Klee ML, Dieter S, Sauer U, Sommer WH. The neurometabolic fingerprint of excessive alcohol drinking. Neuropsychopharmacology, 2015, 40(5):12591268. https://doi.org/10.1038/npp.2014.312 PMID: 25418809 PMCID: PMC4367471

[3] Montesinos J, Alfonso-Loeches S, Guerri C. Impact of the innate immune response in the actions of ethanol on the central nervous system. Alcohol Clin Exp Res, 2016, 40(11):22602270. https://doi.org/10.1111/acer.13208 PMID: 27650785

[4] Skuja S, Groma V, Ravina K, Tarasovs M, Cauce V, Teteris O. Protective reactivity and alteration of the brain tissue in alcoholics evidenced by SOD1, MMP9 immunohistochemistry, and electron microscopy. Ultrastruct Pathol, 2013, 37(5):346355. https://doi.org/10.3109/01913123.2013.810688 PMID: 23875973

[5] Sutherland GT, Sheedy D, Sheahan PJ, Kaplan W, Kril JJ. Comorbidities, confounders, and the white matter transcriptome in chronic alcoholism. Alcohol Clin Exp Res, 2014, 38(4):9941001. https://doi.org/10.1111/acer.12341 PMID: 24460866 PMCID: PMC3984382

[6] de la Monte SM, Kril JJ. Human alcohol-related neuropathology. Acta Neuropathol, 2014, 127(1):71-90. https://doi.org/10.1007/ s00401-013-1233-3 PMID: 24370929 PMCID: PMC4532397

[7] Lippai D, Bala S, Csak T, Kurt-Jones EA, Szabo G. Chronic alcohol-induced microRNA-155 contributes to neuroinflammation in a TLR4-dependent manner in mice. PLoS One, 2013, 8(8):e70945. https://doi.org/10.1371/journal.pone.0070945 PMID: 23951048 PMCID: PMC3739772

[8] Fernandez-Lizarbe S, Montesinos J, Guerri C. Ethanol induces TLR4/TLR2 association, triggering an inflammatory response in microglial cells. J Neurochem, 2013, 126(2):261-273. https:// doi.org/10.1111/jnc.12276 PMID: 23600947

[9] Coleman LG Jr, Zou J, Crews FT. Microglial-derived miRNA let-7 and HMGB1 contribute to ethanol-induced neurotoxicity via TLR7. J Neuroinflammation, 2017, 14(1):22. https://doi. org/10.1186/s12974-017-0799-4 PMID: 28118842 PMCID: PMC5264311

[10] Pomrenze MB, Giovanetti SM. DREADD to the core. Commentary on Purohit et al. (2018): Pharmacogenetic manipulation of the nucleus accumbens alters binge-like alcohol drinking in mice. Alcohol Clin Exp Res, 2018, 42(9):1623-1626. https://doi.org/ 10.1111/acer.13822 PMID: 29957848

[11] Cloninger CR. Etiologic factors in substance abuse: an adoption study perspective. NIDA Res Monogr, 1988, 89:52-72. PMID: 3147398

[12] Cloninger CR. The psychobiological regulation of social cooperation. Nat Med, 1995, 1(7):623-625. https://doi.org/ 10.1038/nm0795-623 PMID: 7585135

[13] Kärkkäinen O, Kupila J, Häkkinen M, Laukkanen V, Tupala E, Kautiainen $\mathrm{H}$, Tiihonen J, Storvik M. AMPA receptors in postmortem brains of Cloninger type 1 and 2 alcoholics: a wholehemisphere autoradiography study. Psychiatry Res, 2013, 214(3):429-434. https://doi.org/10.1016/j.pscychresns.2013. 09.012 PMID: 24119876

[14] Sutherland GT, Sheedy D, Kril JJ. Using autopsy brain tissue to study alcohol-related brain damage in the genomic age. Alcohol Clin Exp Res, 2014, 38(1):1-8. https://doi.org/10.11 11/acer.12243 PMID: 24033426 PMCID: PMC3867579

[15] Brion M, de Timary P, Pitel AL, Maurage P. Source memory in Korsakoff syndrome: disentangling the mechanisms of temporal confusion. Alcohol Clin Exp Res, 2017, 41(3):596607. https://doi.org/10.1111/acer.13318 PMID: 28160301

[16] Kamat PK, Mallonee CJ, George AK, Tyagi SC, Tyagi N. Homocysteine, alcoholism, and its potential epigenetic mechanism. Alcohol Clin Exp Res, 2016, 40(12):2474-2481. https://doi.org/ 10.1111/acer.13234 PMID: 27805256 PMCID: PMC5133158 
[17] Nie X, Wang W, Wang Q, Zhu D, Song H. Intranasal erythropoietin ameliorates neurological function impairments and neural pathology in mice with chronic alcoholism by regulating autophagy-related Nrf2 degradation. Mol Med Rep, 2019, 19(2):1139-1149. https://doi.org/10.3892/mmr.2018.9706 PMID: 30535439 PMCID: PMC6323205

[18] Pascual M, Pla A, Miñarro J, Guerri C. Neuroimmune activation and myelin changes in adolescent rats exposed to high-dose alcohol and associated cognitive dysfunction: a review with reference to human adolescent drinking. Alcohol Alcohol, 2014, 49(2):187-192. https://doi.org/10.1093/alcalc/agt164 PMID: 24217958

[19] Ruiz SM, Oscar-Berman M, Sawyer KS, Valmas MM, Urban T, Harris GJ. Drinking history associations with regional white matter volumes in alcoholic men and women. Alcohol Clin Exp Res, 2013, 37(1):110-122. https://doi.org/10.1111/j.1530-0277. 2012.01862.x PMID: 22725728 PMCID: PMC3459287

[20] Sawyer KS, Oscar-Berman M, Barthelemy OJ, Papadimitriou GM, Harris GJ, Makris N. Gender dimorphism of brain reward system volumes in alcoholism. Psychiatry Res Neuroimaging, 2017, 263:15-25. https://doi.org/10.1016/j.pscychresns.2017.03.001 PMID: 28285206 PMCID: PMC5415444

[21] Trifu S, Vladuti A, Popescu A. Neuroendocrine aspects of pregnancy and postpartum depression. Acta Endocrinol (Bucharest), 2019, 15(3):410-415. https://doi.org/10.4183/ aeb.2019.410 PMID: 32010366 PMCID: PMC6992410

[22] Folescu R, Zamfir CL, Sişu AM, Motoc AGM, Ilie AC, Moise M. Histopathological and imaging modifications in chronic ethanolic encephalopathy. Rom J Morphol Embryol, 2014, 55(3):797801. PMID: 25329105

[23] Lee JH, Heo SH, Chang DI. Early-stage alcoholic cerebellar degeneration: diagnostic imaging clues. J Korean Med Sci, 2015, 30(11):1539. https://doi.org/10.3346/jkms.2015.30.11. 1539 PMID: 26848494 PMCID: PMC4630466

[24] Finegersh A, Homanics GE. Acute ethanol alters multiple histone modifications at model gene promoters in the cerebral cortex. Alcohol Clin Exp Res, 2014, 38(7):1865-1873. https://doi.org/ 10.1111/acer.12465 PMID: 24942484 PMCID: PMC4107049
[25] Trifu SC, Vlăduţi A, Trifu Al. Genetic aspects in schizophrenia. Receptoral theories. Metabolic theories. Rom J Morphol Embryol, 2020, 61(1):25-32. https://doi.org/10.47162/RJME. 61.1.03 PMID: 32747892

[26] Frischknecht U, Hermann D, Tunc-Skarka N, Wang GY, Sack M, van Eijk J, Demirakca T, Falfan-Melgoza C, Krumm B, Dieter S, Spanagel R, Kiefer F, Mann KF, Sommer WH, Ende G, Weber-Fahr W. Negative association between MR-spectroscopic glutamate markers and gray matter volume after alcohol withdrawal in the hippocampus: a translational study in humans and rats. Alcohol Clin Exp Res, 2017, 41(2):323333. https://doi.org/10.1111/acer.13308 PMID: 28098946

[27] Trifu S, Trifu AD. Receptor profiles of atypical antipsychotic molecules. UPB Sci Bull Ser B Chem Mater Sci, 2020, 82(1): 113-128. https://www.scientificbulletin.upb.ro/rev docs arhiva/ full3f8_771428.pdf

[28] Papp-Peka A, Tong M, Kril JJ, De La Monte SM, Sutherland GT. The differential effects of alcohol and nicotine-specific nitrosamine ketone on white matter ultrastructure. Alcohol Alcohol, 2017, 52(2):165-171. https://doi.org/10.1093/alcalc/agw067 PMID: 28182194 PMCID: PMC6075461

[29] Tong M, Yu R, Silbermann E, Zabala V, Deochand C, de la Monte SM. Differential contributions of alcohol and nicotinederived nitrosamine ketone (NNK) to white matter pathology in the adolescent rat brain. Alcohol Alcohol, 2015, 50(6):680689. https://doi.org/10.1093/alcalc/agv102 PMID: 26373813 PMCID: PMC4608624

[30] Paulus W. Lifestyle neuropathology: how our behavior harms our brains and what we can do about it. Acta Neuropathol, 2014, 127(1):1. https://doi.org/10.1007/s00401-013-1234-2 PMID: 24362442

[31] Trifu S. Neuroendocrine insights into burnout syndrome. Acta Endocrinol (Bucharest), 2019, 15(3):404-405. https:// doi.org/10.4183/aeb.2019.404 PMID: 32010363 PMCID: PMC6992409

\section{Corresponding author}

Ana Miruna Drăgoi, Department of Psychiatry, Prof. Dr. Alexandru Obregia Clinical Hospital for Psychiatry, 10 Berceni Highroad, Sector 4, 041914 Bucharest, Romania; Phone +40770-420 108, e-mail: anamirunadragoi@gmail.com

Received: February 1, 2020

Accepted: October 22, 2020 